\title{
Giant homopolymers with amphiphilicity
}

A novel class of amphiphilic dendronized homopolymers has been reported by The Department of Polymer Materials, Shanghai University. By tuning the structures, these polymers can show thermoresponsive properties and adopt ordered secondary structures or self-assemble into ordered porous films. The study is reported in Issue 12 (December 2010) of SCIENCE CHINA Chemistry because of its significant research value.

Most amphiphilic polymers are block copolymers, and amphiphilic block copolymers have attracted intense research attention in recent decades because of their ability to form versatile superstructures via self-assembly. Although it is much easier to synthesize homopolymers than block copolymers (which are normally synthesized via living polymerization), amphiphilic homopolymers have received little attention until now. Amphiphilic homopolymers contain both hydrophilic and hydrophobic moieties in each repeating unit, and this construction may lead to special assembly mechanisms.

Dendronization of a linear polymer reduces the attainable backbone conformations and transforms a random coil polymer into a cylindrically shaped molecular object, and thus leads to the formation of a novel class of nano-sized polymers referred to as dendronized polymers. Combination of the structural characteristics of amphiphilic homopolymers with dendronized polymers is promising for developing a novel class of giant macromolecules with sizable hydrophilic and hydrophobic moieties in each repeating unit.

In the present work, the authors designed and synthesized three types of novel amphiphilic homopolymers based on the dendronized polymer concept. Hydrophilic oligo(ethylene glycol) (OEG)-based and hydrophobic proline-based dendrons were introduced into one repeating unit to form amphiphilic macromonomers cored by a chiral lysinol unit. The corresponding polymers were obtained via free radical polymerization of the macromonomers. All polymers consisted of first generation OEG-based dendrons with proline-based dendrons of different generation, which afforded polymers with variable amphiphilicity. Two of the polymers are water soluble at room temperature and show specific thermoresponsiveness at elevated temperatures. The most hydrophobic polymer is not water soluble but can self-assemble into highly ordered porous films via the "breath figure" technique. All of these polymers adopt ordered helical conformations in solution.

One journal reviewer noted: "This paper emphasizes the efficient synthesis and characterization of a novel class of amphiphilic dendronized homopolymers. The result is of academic and practical significance". Another reviewer commented: "It expanded the research results on dendronized polymers and also on amphiphilic homopolymers. It offered us a new principle to develop novel amphiphilic polymer structures", A series of papers on stimuli-responsive dendronized polymers written by Dr. Li and Prof. Zhang have already been published in various international journals.

The authors are affiliated with the Laboratory of Polymer Synthesis, SHU. This laboratory is conducting research mainly in four directions: stimuli-responsive materials, dendronized polymers, polymer brushes and polypeptides. This work was supported by the National Natural Science Foundation of China (20374047 and 20574062), ETH Research (ETH-1608-1) and the Swiss National Science Foundation (200021-113690).

See the article: Li W, Zhang A. Amphiphilic dendronized homopolymers. Sci China Chem, 2010, 53: 2509-2519

Open Access This article is distributed under the terms of the Creative Commons Attribution License which permits any use, distribution, and reproduction in any medium, provided the original author(s) and source are credited. 